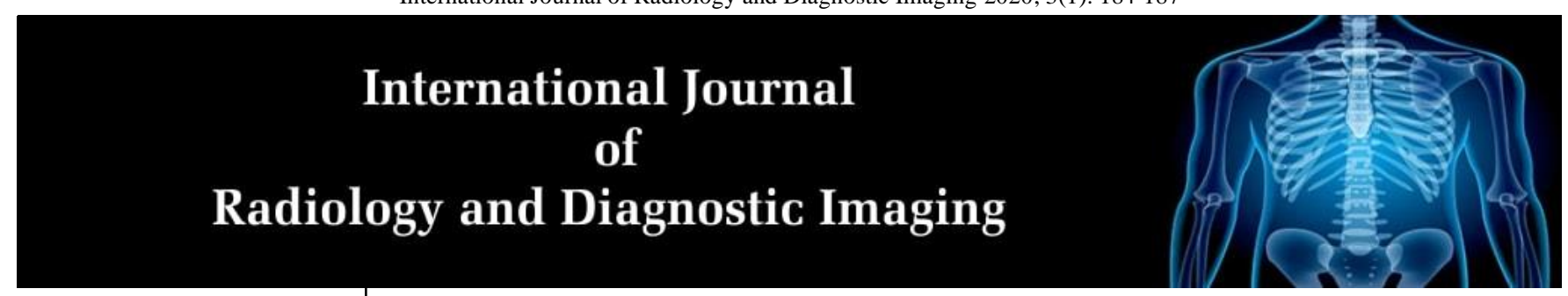

E-ISSN: 2664-4444

P-ISSN: 2664-4436

IJRDI 2020; 3(1): 184-187

Received: 04-11-2019

Accepted: 07-12-2019

Dr. Raghavendra Temkar V Assistant Professor,

Department of Radiology, Sambhram Institute of

Medical Sciences and Research, KGF, Karnataka, India

Dr. Bagyam Raghavan Senior Consultant Radiologist, Department of Radiology, Apollo Speciality Hospital, Chennai, Tamil Nadu, India
Corresponding Author: Dr. Raghavendra Temkar V Assistant Professor, Department of Radiology, Sambhram Institute of Medical Sciences and Research, KGF, Karnataka, India

\section{Clinical profile of patients subjected for DCE-MRI and DWI for suspicious breast lesions}

\author{
Dr. Raghavendra Temkar V and Dr. Bagyam Raghavan
}

DOI: http://dx.doi.org/10.33545/26644436.2020.v3.i1c.70

\begin{abstract}
In India, it is the second most common cancer after carcinoma cervix. However, its incidence is rising and it has already surpassed the incidence of carcinoma cervix in metros like Delhi with age adjusted incidence of 29.0 per 100,000 per year in the year 2003. Breast cancer is a major health issue in women and the stage (extent of a cancer in the body) of the cancer at the time of diagnosis, determines the treatment options and also has a strong influence on the length of survival. All the patients were selected according to the inclusion and exclusion criteria. Informed consent was taken from each patient included in the study. Breast MR imaging was performed within days 7-14 of the menstrual cycle for premenopausal women. A thorough clinical history was taken. All the patients were explained about the procedure to make them comfortable. Various indications for which the patients were referred for MRI of the breast in our study were as follows: mass (51 Of 62 patients; 82.2\%) detected in mammogram, ultrasound or clinical examination; focal asymmetry on mammogram (4 of 62 patients; 6.5\%); $2(3.2 \%)$ patients had bloody nipple discharge; $2(3.2 \%)$ patients presented with mastalgia; 2 (3.2\%) patients had nipple eczema; one (1.6\%) patient had breast swelling.
\end{abstract}

Keywords: DCE-MRI, DWI, breast lesions

\section{Introduction}

Breast is a modified apocrine sweat gland. Diseases of breast have attracted medical interest since as long ago 3000 B.C. In the advanced civilization of Egypt during the age of pyramids (3000-2500 B.C.) several cases of women with tumours of the breast have been described. It is probable that malignant tumours of the female breast were the first human cancers which were identified and distinguished from other non-malignant diseases ${ }^{[1]}$.

Breast disease is one of the most common complaints of females in any age group and breast masses vary from benign to malignant with varied aetiologies.

Breast carcinoma is one of the common malignant diseases among women in the world. According to the International Agency for Research on Cancer (IARC), there are approximately 1.2 million new diagnoses of worldwide breast carcinoma every year and 410,000 are followed with death ${ }^{[2]}$.

In India, it is the second most common cancer after carcinoma cervix. However, its incidence is rising and it has already surpassed the incidence of carcinoma cervix in metros like Delhi with age adjusted incidence of 29.0 per 100,000 per year in the year $2003^{[3]}$.

Breast cancer is a major health issue in women and the stage (extent of a cancer in the body) of the cancer at the time of diagnosis, determines the treatment options and also has a strong influence on the length of survival.

The radiological examination of breast is an integral part of modern multidisciplinary approach for effective management of breast disease. The aim of the breast imaging is to assess the probability of a lesion being benign or malignant.

Currently screen film X-ray mammography, ultrasound, colour Doppler and MRI are being utilised for detection of breast cancer.

Compared with x-ray mammography and breast ultrasound, contrast enhanced MRI is a breast imaging technique that offers information on morphological and functional features such as tissue perfusion and enhancement kinetics ${ }^{[4]}$.

Although MRI has high sensitivity in the diagnosis of breast cancer, as high as $89-100 \%$ for invasive cancers, the specificity is moderate (72\% overall specificity in 44 studies) and specificity differs widely based on the criteria used for diagnosing malignant lesions from benign lesions ${ }^{[5]}$. 
Over the past decade newer MR imaging and analysing techniques are being constantly developed in order to increase the specificity and positive predictive value (PPV) of breast MRI. One such newer advanced technique is diffusion weighted magnetic resonance imaging (DW-MRI). DW MRI provides information on tissue microstructure and diffusivity of water molecules which are shown to be an important index of tumour grade and local tissue composition, which help in the early and sensitive detection of abnormality ${ }^{[6]}$.

DW MRI was designed to reflect and measure the diffusivity of water molecules within the biological tissues and to quantify such movement with apparent diffusion coefficient (ADC) value.

The advances in MR imaging techniques allow for the possibility to improve the diagnostic performance by combining the advantages of different imaging techniques. Such combination of imaging techniques are promising in clinical diagnosis by decreasing the inter-observer bias in interpretation of the images and by shortening the diagnosis time. Combining morphological characteristics with enhancement kinetics can improve the interpretation and diagnostic performance of breast lesion. However, there is less research on investigation of the combined performance of both DCE MRI and DWI in discrimination of breast masses.

This inadequate evidence available in the literature necessitates the application of DWI in routine breast MR imaging protocols so as to improve the diagnostic accuracy of breast MRI.

Diffusion weighted imaging (DWI) is an important resource as an adjunctive tool to breast MRI to differentiate benign from malignant lesions. This sequence can be added easily to the routine standard breast magnetic resonance imaging sequences and thereby facilitate early and accurate detection of the breast diseases specially carcinomas.

\section{Methodology}

All the patients were selected according to the inclusion and exclusion criteria. Informed consent was taken from each patient included in the study. Breast MR imaging was performed within days 7-14 of the menstrual cycle for premenopausal women.

A thorough clinical history was taken. All the patients were explained about the procedure to make them comfortable.

MR examination was performed on a 1.5 Tesla Philips achieva MR scanner (Philips medical system, Netherlands) using a dedicated 8 channel bilateral breast coil with patients positioned prone and the breasts hanging freely into the cushioned openings which are surrounded by the specialized breast coil, which is a signal receiver and works with the MRI unit to create images.

All the patients were imaged with dynamic contrast enhanced MRI and diffusion weighted MRI.

All the MRI images were interpreted using the American College of Radiology BI-RADS breast MRI lexicon incorporating morphologic and kinetic features. The palpable, sonographic or mammographically suspicious lesion was considered the index lesion. The index lesion was identified on subtracted and T2-weighted images. In patients with multiple lesions in one breast with similar MR imaging features, the lesion with the most suspicious MR features was considered as the index lesion. The MR images were analyzed for the morphologic features such as size, shape, signal intensity and enhancement pattern. On dynamic MR Images, a region of interest (ROI) was placed at the most enhancing area of the lesion and time signal intensity curves were obtained.

The index lesion was classified as either mass (threedimensional space-occupying lesion) or non-mass like enhancement and morphologic features were analyzed. For the masses, shape, margins, type of enhancement and signal intensity on T1- and T2-weighted images were analysed. On $\mathrm{T} 1 \mathrm{w}$ and $\mathrm{T} 2 \mathrm{w}$ images masses were considered hypointense, hyperintense, isointense and heterointense relative to adjacent breast glandular parenchyma. The nonmass lesions were assessed for the distribution, internal enhancement pattern, and symmetry.

The dynamic scans were assessed for the degree of enhancement and time signal intensity curves which were acquired with the help of dedicated software on computer. The enhancement kinetics of the lesions were analysed based on two standpoints. First is the peak percentage increase of signal intensity in the early phase, (the first two contrast-enhanced acquisitions-wash-in rate) and the second is the shape of the curve after the early phase (wash- out kinetics). A wash-in rate of more than $80 \%$ was defined as fast or strong, between $50 \%$ and $80 \%$ as intermediate, and less than $50 \%$ as slow enhancement.

Three types of time signal intensity curves were obtained. Type-I (progressive) curve-enhancement continues to increase with each post contrast sequence. Type-II (plateau) curves; enhancement levels off after the first post contrast sequence. In the Type-III (washout) curve; enhancement decreases after initial rise. These curves were obtained by placing the region of interest (ROI) on the lesion.

In the morphologic analysis of the MR images, oval or round shaped masses or masses with smooth margins; presence of fatty signal within the lesion on $\mathrm{T} 1$ and $\mathrm{T} 2$ images, homogenous enhancement with non-enhancing internal septation, bilateral symmetric non- mass enhancement in any distribution; and unilateral, asymmetric non mass enhancements in local or regional distribution were considered negative for malignancy in our study.

Patients with benign findings at MR imaging were followed-up within 1 year with mammography or ultrasound to ensure the stability of the lesion.

Irregular shaped masses with irregular or spiculated margins, heterogeneous or rim enhancement, and clumped type of non-mass like enhancement in ductal or segmental distribution were considered positive for malignancy.

In the kinetic analysis of the MR images, type-I curve (slow initial enhancement and persistent increase in uptake) was considered negative for malignancy. Type II and III curves (strong early enhancement with an ensuing plateau or washout time) were considered positive for malignancy.

\section{Results}

Table 1: Distribution of patients according to age group ( $n=62)$.

\begin{tabular}{|c|c|c|}
\hline Age Groups (in years) & Number of Patients & Percentage (\%) \\
\hline $21-30$ & 2 & 3.22 \\
\hline $31-40$ & 15 & 24.19 \\
\hline $41-50$ & 23 & 37.09 \\
\hline $51-60$ & 15 & 24.19 \\
\hline $61-70$ & 7 & 11.29 \\
\hline
\end{tabular}


Majority of the patients were in the fifth decade of life $(n=23)$. There were equal number of patients in the both Fourth and Sixth decade $(n=15)$. Seven patients were above 60 years and two patients were below 30 years. The median age was 47 years (range $=23-70$ years).

Table 2: Clinical and mammogram features for which patients were referred for breast MRI.

\begin{tabular}{|c|c|c|}
\hline Symptoms & Number of patients & Percentage (\%) \\
\hline Mass & 51 & 82.2 \\
\hline Focal asymmetry & 4 & 6.5 \\
\hline Nipple discharge & 2 & 3.2 \\
\hline Mastalgia & 2 & 3.2 \\
\hline Nipple eczema & 2 & 3.2 \\
\hline Swelling & 1 & 1.6 \\
\hline
\end{tabular}

Various indications for which the patients were referred for MRI of the breast in our study were as follows: mass (51 0f 62 patients; $82.2 \%$ ) detected in mammogram, ultrasound or clinical examination; focal asymmetry on mammogram (4 of 62 patients; $6.5 \%) ; 2$ (3.2\%) patients had bloody nipple discharge; $2(3.2 \%)$ patients presented with mastalgia; 2 (3.2\%) patients had nipple eczema; one (1.6\%) patient had breast swelling.

Table 3: Distribution of laterality of the index lesion.

\begin{tabular}{|c|c|c|}
\hline Laterality & Frequency & Percentage \\
\hline Right & 41 & 66.1 \\
\hline Left & 21 & 33.9 \\
\hline Total & 62 & 100 \\
\hline
\end{tabular}

In the present study, $66.1 \%$ of index lesions were found in the right breast and $33.9 \%$ were found in the left breast.

Table 4: Breast parenchymal composition.

\begin{tabular}{|c|c|c|}
\hline Composition & Frequency & Percentage (\%) \\
\hline Almost entirely fat & 4 & 6.5 \\
\hline Fatty with scattered fibroglandular tissue & 28 & 45.2 \\
\hline Heterogenous fibroglandular tissue & 29 & 46.8 \\
\hline Extremely fibroglandular tissue & 1 & 1.6 \\
\hline Total & 62 & 100.0 \\
\hline
\end{tabular}

Of the 62 patients, the breast parenchymal composition were as follows; $4(6.5 \%)$ had predominantly fatty composition; $28(45.2 \%)$ had fatty with scattered glandular tissue; 30 $(48.4 \%)$ had glandular parenchymal composition.

The mean size of the benign and malignant lesions in our study was $1.44 \pm 0.40 \mathrm{cms}$ and $2.47 \pm 1.67 \mathrm{cms}$ respectively.

In our study, the minimum and maximum sizes of benign lesions were $1.0 \mathrm{cms}$ and $2.5 \mathrm{cms}$. The minimum and maximum sizes of malignant lesions were $1.0 \mathrm{cms}$ and 9.70 cms.

Table 5: Shape of Index Lesions on MRI $(n=58)$.

\begin{tabular}{|c|c|c|c|}
\hline Shape & Malignant & Benign & Total \\
\hline Oval & $0 / 40(0 \%)$ & $11 / 18(61.1 \%)$ & $11 / 58(18.9 \%)$ \\
\hline Round & $0 / 40(0 \%)$ & $4 / 18(22.2 \%)$ & $4 / 58(6.8 \%)$ \\
\hline Irregular & $40 / 40(100 \%)$ & $3 / 18(16.7 \%)$ & $43 / 58(74.1 \%)$ \\
\hline
\end{tabular}

In our study, the most common shape of the malignant index lesions was irregular $(n=40)$. Three of the benign lesions showed irregular shape. The most common shape of the benign index lesions was oval $(n=11)$ followed by round $(n=4)$.
Table 6: Margins of Index Lesions on MRI $(n=58)$.

\begin{tabular}{|c|c|c|c|}
\hline Margins & Malignant & Benign & Total \\
\hline Smooth & $0 / 40(0 \%)$ & $15 / 18(83.3 \%)$ & $15 / 58(25.8 \%)$ \\
\hline Irregular & $17 / 40(42.5 \%)$ & $3 / 18(16.6 \%)$ & $20 / 58(34.5 \%)$ \\
\hline Spiculated & $23 / 40(57.5 \%)$ & $0 / 18(0 \%)$ & $23 / 58(39.6 \%)$ \\
\hline
\end{tabular}

In our study, majority of the malignant lesions detected on MRI had spiculated $(\mathrm{n}=23, \quad 57.5 \%)$ or irregular margins $(\mathrm{n}=17,42.5 \%)$. No malignant lesion showed smooth margin. Most of the benign lesions detected on MRI showed smooth margins $(n=15,83.3 \%)$ and three of the benign lesions showed irregular margins and histopathological diagnosis for these lesions were chronic inflammation, fat necrosis and intraductal papillary lesion. None of the benign lesions had spiculated margin.

Table 7: Signal Intensity Characteristics of Index Mass Lesions on T1WI $(n=62)$.

\begin{tabular}{|c|c|c|c|}
\hline $\begin{array}{c}\text { Signal intensity on T1 } \\
\text { weighted images }\end{array}$ & Malignant & Benign & Total \\
\hline Hypointense & $40 / 42(95.2 \%)$ & $19 / 20(95 \%)$ & $59 / 62(95.2 \%)$ \\
\hline Isointense & $1 / 42(2.4 \%)$ & $0 / 20(0 \%)$ & $1 / 62(1.6 \%)$ \\
\hline Hyperintense & $1 / 42(2.4 \%)$ & $1 / 20(5 \%)$ & $2 / 62(3.2 \%)$ \\
\hline
\end{tabular}

\section{Discussion}

Breast cancer is a major source of morbidity and mortality in women. Breast MRI is now increasingly being used to accurately diagnose breast cancers, and as a problem solving tool in cases in which mammography and breast ultrasound are inconclusive or discrepant. Multipara metric breast MRI included assessment of lesion morphology and kinetic features, using high spatial resolution and dynamic MRI. This resulted in good sensitivity but the specificity remained moderate. To improve the specificity of breast MRI, other adjunctive techniques such as diffusion-weighted imaging, T2*-weighted perfusion imaging and MR spectroscopy are being studied. And also diffusion weighted imaging was proposed to be included in the 5th edition of BIRADS as a part of multiparametric breast MR imaging. Currently more prospective data is needed to validate these newer MR imaging technique.

In our prospective observational study, 62 patients with breast lesions underwent breast MRI with a protocol combining dynamic contrast enhanced MR and diffusion weighted imaging in order to improve the specificity of breast MRI.

Breast lump was the most common presenting complaint (50 0f 62 patients; $80.6 \%$ ) in our study. Other presentations included four patients with focal asymmetry on mammogram, two patients with serosanguinous nipple discharge, two with mastalgia and two patients had nipple eczema. Our study observations were consistent with the previous studies by Mumtaz et al., ${ }^{[7]}$; Berg et al., ${ }^{[8]}$ and Liberman et al., ${ }^{[9]}$ who also reported breast lump as the common presenting complaint in breast cancer.

Morphological characteristics were observed in all the lesions. All the malignant lesions $(n=41 / 41 ; 100 \%)$ showed either irregular or spiculated margins. No malignant lesion had smooth margin. These findings were similar to previous studies by Liberman et al., ${ }^{[9]}$ and Kim et al., ${ }^{[10]}$ who also reported irregular shape as the most common type, followed by lobulated margins.

Majority of the benign lesions in our study $(n=15 / 18$; $83.3 \%$ ) had oval or round shape with smooth margins which 
was consistent with study by Nunes et al., ${ }^{[11]}$ in which smooth (38/40) and lobulated (84/93) margins were the common findings in benign breast lesions. Christiania Kuhl, who also described the appearance of benign lesions as focal mass with round or oval shape and smooth margins.

In our study, $52.38 \%$ of the malignant tumours had T2 hyper intensity, while $42.85 \%$ showed heterointense signal on $\mathrm{T} 2 \mathrm{w}$ images. $4.76 \%$ of the malignant tumours were hypointense. Majority (75\%) of the benign tumours had T2 hyper intensity, $15 \%$ had heterointense signal and $10 \%$ showed hypointense signal on T2w images.

Gorane Santamaría et al., [12] have studied the histopathological characters of the benign and malignant breast tumours and have described the various components in the tumours which can produce $\mathrm{T} 2$ hyper intensity on breast magnetic resonance (MR) imaging. Tumours with profound necrosis, a cystic or micro cystic component, intratumoural fat or sebaceous component, stromal components like mucin, loose myxoid tissue, oedema and intratumoural hemorrhagic changes may appear hyper intense on $\mathrm{T} 2 \mathrm{w}$ images.

In our study, invasive ductal carcinomas formed the majority of the malignant tumours. Invasive ductal carcinomas are the most common type of the invasive malignant breast tumors accounting for about $65 \%-80 \%$. Cystic changes within invasive ductal carcinomas though uncommon may occur as a result of necrosis with accompanying hemorrhage in the degenerated areas. Many of the invasive ductal carcinomas may appear hyper intense on T2-weighted images. Tsuda et al. described that highergrade invasive ductal carcinomas containing large central acellular zone appear hyper intense on T2w images. And also tumors with extensive necrosis are hyper intense on $\mathrm{T} 2 \mathrm{w}$ images.

$10 \%-14 \%$ of invasive breast carcinomas are invasive lobular carcinomas. Literature data on $\mathrm{T} 2 \mathrm{w}$ imaging findings of invasive lobular carcinoma is limited. Histologically, there is lack of cohesiveness of the cells in the invasive lobular carcinomas. Gorane Santamaría et al., suggested that this loose stromal tissue within invasive lobular carcinomas may be the cause of T2 hyper intensity.

\section{Conclusion}

- The mean size of the benign and malignant lesions in our study was $1.44 \pm 0.40 \mathrm{cms}$ and $2.47 \pm 1.67 \mathrm{cms}$ respectively.

- In our study, the minimum and maximum sizes of benign lesions were $1.0 \mathrm{cms}$ and $2.5 \mathrm{cms}$. The minimum and maximum sizes of malignant lesions were $1.0 \mathrm{cms}$ and $9.70 \mathrm{cms}$.

- Majority of the malignant lesions detected on MRI had speculated $(n=23,57.5 \%)$ or irregular margins $(n=17$, $42.5 \%$ ). No malignant lesion showed smooth margin. Most of the benign lesions detected on MRI showed smooth margins $(n=15,83.3 \%)$ and three of the benign lesions showed irregular margins.

\section{References}

1. Huang W, Fisher PR, Dulaimy K, Tudorica LA, O'Hea $\mathrm{B}$ et al. Detection of breast malignancy: diagnostic MR protocol for improved specificity. Radiology. 2004; 232(2):585-91.

2. Heywang-Köbrunner SH, Viehweg P, Heinig A, Kuchler C. Contrast enhanced MRI of the breast: accuracy, value, controversies, solutions. Eur J Radiol. 1997; 24(2):94-108.

3. Schnall MD, Rosten S, Englander S, Orel SG, Nunes LW. A combined architectural and kinetic interpretation model for breast MR images. Acad Radiol. 2001; 8(7):591-7.

4. Kinkel K, Helbich TH, Esserman LJ, Barclay J, Schwerin EH, Sickles EA et al. Dynamic high-spatialresolution MR imaging of suspicious breast lesions: diagnostic criteria and interobserver variability. AJR Am J Roentgenol. 2000; 175(1):35-43.

5. Warren RM, Pointon L, Thompson D, Hoff R, Gilbert FJ, Padhani A et al; UK Magnetic Resonance Imaging in Breast Screening (MARIBS) Study Group. Reading protocol for dynamic contrast-enhanced MR images of the breast: sensitivity and specificity analysis. Radiology. 2005; 236(3):779-88.

6. Peters NH, Borel Rinkes IH, Zuithoff NP, Mali WP, Moons KG, Peeters PH. Meta-analysis of MR imaging in the diagnosis of breast lesions. Radiology. 2008; 246(1):116-24.

7. Mumtaz H, Hall-Craggs MA, Davidson T, Walmsley $\mathrm{K}$, Thurell W, Kissin MW et al. Staging of symptomatic primary breast cancer with MR imaging. AJR Am J Roentgenol. 1997; 169(2):417-24.

8. Berg WA, Gutierrez L, NessAiver MS, Carter WB, Bhargavan M, Lewis RS et al. Diagnostic accuracy of mammography, clinical examination, US, and MR imaging in preoperative assessment of breast cancer. Radiology. 2004; 233(3):830-49.

9. Liberman L, Morris EA, Dershaw DD, Abramson AF, Tan LK. MR imaging of the ipsilateral breast in women with percutaneously proven breast cancer. AJR Am J Roentgenol. 2003; 180(4):901-10.

10. Kim SJ, Morris EA, Liberman L, Ballon DJ, La Trenta LR, Hadar $\mathrm{O}$ et al. Observer variability and applicability of BI-RADS terminology for breast MR imaging: invasive carcinomas as focal masses. AJR Am J Roentgenol. 2001; 177(3):551-7.

11. Nunes LW, Schnall MD, Orel SG. Update of breast MR imaging architectural interpretation model. Radiology. 2001; 219(2):484-94.

12. Santamaría G, Velasco M, Bargalló X, Caparrós X, Farrús B, Luis Fernández P. Radiologic and pathologic findings in breast tumors with high signal intensity on T2-weighted MR images. Radiographics. 2010; 30(2):533-48. 